\title{
EDITORIAL \\ Guest Editorial: Special Issue on Algorithms and Systems on Big Graph Processing
}

\author{
Qiang-Sheng Hua ${ }^{1} \cdot$ Xuanhua Shi $^{1} \cdot$ Yinglong Xia $^{2} \cdot$ Howie Huang ${ }^{3}$ \\ Published online: 30 April 2019 \\ (c) Springer Science+Business Media, LLC, part of Springer Nature 2019
}

The graph is an important data structure; it can be used to model many practical problems and is widely studied in various fields, such as social networks, informatics and many others. With the explosive growth of information in recent years, as a data structure, the scale of the graph is also getting bigger and bigger. The original graph processing systems and algorithms are no longer applicable. So how to process big graphs has become a research hotspot.

The aim of this special issue is to provide an introduction to the burgeoning topic of algorithms and systems on big graph processing. Undergoing a rigorous selection process, seven papers are accepted in this special issue. Among these seven papers, two papers discuss the algorithms and systems on big graph processing, two papers present methods of protecting privacy, and the other three papers argue some applications and extensions of big graphs.

Parallel processing of the big graphs such as social networks, Web graphs and so on is a significant hotspot nowadays. However, in the graph processing frameworks based on GPU, since there is frequent data exchange between host and GPU, the performance of iterative traversing-based graph algorithms is seriously affected. The first article titled "WolfPath: Accelerating iterative traversing-based graph processing algorithms on GPU" by Zhu et al. introduces a new data structure to represent the graph, which helps greatly reduce the frequency of data exchange. When there is not enough main memory to process big graphs datasets, the out-of-core graph processing systems will be useful. Most of these systems execute in synchronous rounds to adapt to the iterative nature of graph algorithms. These systems usually sequentially scan the whole graph dataset in each iteration for fully utilizing the disk bandwidth, which

Qiang-Sheng Hua

qshua@hust.edu.cn

1 Services Computing Technology and System Lab/Big Data Technology and System Lab/Cluster and Grid Computing Lab, School of Computer Science and Technology, Huazhong University of Science and Technology, Wuhan, People's Republic of China

2 Huawei Research America, Santa Clara, USA

3 Department of Electrical and Computer Engineering, The George Washington University, Washington, USA 
may cause large I/O overhead. The second article titled "BlockGraphChi: Enabling Block Update in Out-of-core Graph Processing" by Shao et al. studies the use of block updating in a typical out-of-core GraphChi graph system. The block update model can reduce the number of iterations of graph traversal algorithms.

The research of social networks is a hotspot in the field of complex networks and parallel processing. It focuses on the interactions and connections among individuals. From a mathematical point of view, social networks can be modeled by big graphs. There are some challenges of social networks to solve, such as the lack of effective supervision from the system and the uncooperative behaviors. So how to design a system whose individuals can cooperate is a hot topic in social networks. The third paper titled "Analysis for Behavioral Economics in Social Networks: An Altruism-Based Dynamic Cooperation Model" by Li et al. introduces the reciprocal altruism theory into the incentive mechanism. The connection strengths between two nodes depend on whether the two nodes cooperate or not. And the paper proposes an Altruism-Based Dynamic Model(ABDM) in social networks which is proved to be effective by simulations. Soundness is an important requirement of a system. It guarantees no deadlock and livelock and each activity has potential to be executed in the system. The paper titled "Soundness Analytics of Composed Logical Workflow Nets" by Liu et al. studies the soundness of composed logical work flow nets by analyzing whether a composed logical workflow net is covered by its composed path nets and whether composed single line nets are included by composed path nets.

With the rapid development of network technology, privacy protection is another challenge to be considered in social networks. Because the information transformed in the system can be utilized to attack the nodes, simple removal of labels of nodes does not work. Previous work generally supposes that the attackers only know the neighbor nodes of target nodes. However, the graph structure with signed edge attributes could cause serious privacy leakage of social networks. The fifth paper titled "Against Signed Graph Deanonymization Attacks on Social Networks" by Gao et al. takes the signed attribute of edges into account when achieving k-anonymity privacy protection for social networks. Intrusion Detection System (IDS) is an innovative and proactive network security technology. There are four main characteristics of intrusion data that affect the performance of IDS, including multicomponent, data imbalance, timevarying and unknown attacks. Based on Hybrid Multi-Level Data Mining, the sixth paper titled "An Intrusion Detection Framework based on Hybrid Multi-Level Data Mining" by Yao et al. proposes a novel IDS framework called HMLD to address these issues.

As a common processing method, query is widely used in many areas, such as graph processing, machine learning and statistics. However, queries are usually priced according to vendor-specified fixed views (API) or number of transactions, which ignores computing resource consumption for query and information that the answer brings. The last paper titled "A Novel Auction-based Query Pricing Schema” by Wang et al. takes both information value and computational cost into account and designs the query auction that is truthful and computationally efficient.

Collectively, these accepted papers illustrate the diverse range of issues such as social networks, privacy protection and big graph processing. And they provide a 
detailed compilation of the diverse range of issues currently being investigated in the field of big graph processing.

Publisher's Note Springer Nature remains neutral with regard to jurisdictional claims in published maps and institutional affiliations. 